OPEN ACCESS

Edited by:

Diana Bahia,

Universidade Federal de Minas Gerais,

Brazil

Reviewed by:

Hridayesh Prakash,

University of Hyderabad, India

David MacHugh,

University College Dublin, Ireland

${ }^{*}$ Correspondence:

Qian Yang

zxbyq@njau.edu.cn

Specialty section:

This article was submitted to

Microbial Immunology,

a section of the journal

Frontiers in Microbiology

Received: 26 September 2016

Accepted: 13 February 2017

Published: 22 March 2017

Citation:

Lin J, Xia J, Tu CZ, Zhang KY, Zeng Y and Yang Q (2017) H9N2 Avian Influenza Virus Protein PB1 Enhances the Immune Responses of Bone Marrow-Derived Dendritic Cells by

Down-Regulating miR375.

Front. Microbiol. 8:287.

doi: 10.3389/fmicb.2017.00287

\section{H9N2 Avian Influenza Virus Protein PB1 Enhances the Immune Responses of Bone Marrow-Derived Dendritic Cells by Down-Regulating miR375}

\author{
Jian Lin ${ }^{1}$, Jing Xia ${ }^{1}$, Chong Z. Tu ${ }^{2}$, Ke Y. Zhang ${ }^{1}$, Yan Zeng ${ }^{1}$ and Qian Yang ${ }^{1 *}$ \\ ${ }^{1}$ Department of Zoology, College of Life Science, Nanjing Agricultural University, Jiangsu, China, ${ }^{2}$ Department of \\ Histoembryology, College of Veterinary Medicine, Nanjing Agricultural University, Jiangsu, China
}

Polymerase basic protein 1 (PB1), the catalytic core of the influenza A virus RNA polymerase complex, is essential for viral transcription and replication. Dendritic cells (DCs) possess important antigen presenting ability and a crucial role in recognizing and clearing virus. MicroRNA (miRNA) influence the development of DCs and their ability to present antigens as well as the ability of avian influenza virus (AIV) to infect host cells and replicate. Here, we studied the molecular mechanism underlying the miRNA-mediated regulation of immune function in mouse DCs. We first screened for and verified the induction of miRNAs in DCs after PB1 transfection. Results showed that the viral protein PB1 down-regulated the expression of miR375, miR146, miR339, and miR679 in DCs, consistent with the results of H9N2 virus treatment; however, the expression of miR222 and miR499, also reduced in the presence of PB1, was in contrast to the results of H9N2 virus treatment. Our results suggest that PB1 enhanced the ability of DCs to present antigens, activate lymphocytes, and secrete cytokines, while miR375 over-expression repressed activation of DC maturation. Nevertheless, PB1 could not promote DC maturation once miR375 was inhibited. Finally, we revealed that PB1 inhibited the P-Jnk/Jnk signaling pathway, but activated the p-Erk/Erk signaling pathway. While inhibition of miR375 -activated the p-Erk/Erk and p-p38/p38 signaling pathway, but repressed the P-Jnk/Jnk signaling pathway. Taken together, results of our studies shed new light on the roles and mechanisms of PB1 and miR375 in regulating DC function and suggest new strategies for combating AIV.

Keywords: H9N2 AIV, PB1, miRNA, dendritic cells, immune regulation

\section{INTRODUCTION}

The influenza virus contains eight segments of a single-stranded RNA genome with negative polarity. The H9N2 subtype avian influenza virus (AIV), classified as a low pathogenic AIV, has high genetic variability and has shown both increases in virulence and ability to cross the host barrier (Peiris et al., 1999; Jin et al., 2014; Shaib et al., 2014; Zhou et al., 2014). Since the H7N9 and 
H10N8 AIV outbreaks in 2013 resulted from recombination between H9N2 and other influenza subtypes, H9N2 AIV is a subject of intense research (Fang et al., 2013; Pu et al., 2015). The virus polymerase complex of H9N2 AIV, consisting of the polymerase basic protein 1 ( $\mathrm{PB} 1)$, polymerase basic protein 2 (PB2) and polymerase acidic protein (PA) subunits, has been reported to charge for catalyzing both viral RNA genome replication and transcription. Previous studies have shown that $\mathrm{PB} 1$ serves as a core subunit to incorporate PA and PB2 into the polymerase complex by directly interacting with PA and PB2 (Hemerka et al., 2009). Recently studies also reveal that PB1 interacted with PA was an attractive target for drug treatment (Massari et al., 2016; Swale et al., 2016).

There is a continuing need for novel anti-influenza therapeutics using new targets or creative strategies and the pathogenicity of a virus is determined not only by its characteristics but also by the host immune response (O'Donnell and Subbarao, 2011). Dendritic cells (DCs) is a professional and effective antigen-presenting cells in the innate immune response (Tucci et al., 2014). Influenza virus is a human pathogen and also naturally infects a large range of animals. Previous studies have found that the mouse model of influenza virus infection is useful for understanding host immune responses and host-pathogen interaction. Zhou found that miR2911, a honeysuckle (HS)-encoded atypical microRNA (miRNA), directly targets Influenza A virus with various subtypes (Zhou et al., 2015). Also, Isakova-Sivak and colleagues have used the mouse model to study the infectivity, immunogenicity and cross-protective efficacy of live attenuated influenza vaccines containing nucleoprotein from cold-adapted or wild-type influenza virus (Isakova-Sivak et al., 2017). Consequently, because human blood and humanized mice were not available for our studies, we developed a suitable animal model to study AIV responses in mammalian DCs. AIV infection affects the maturation, antigen presenting ability, and cytokine secretion of DCs (Lin et al., 2014). The binding of pathogen-associated molecular patterns to receptors expressed by DCs may activate DCs (Lopez et al., 2004; Liang et al., 2013), but it remains unclear how AIVs produce changes in DCs and how DCs respond to AIV infection.

MicroRNA (miRNA) have emerged as key regulators of innate immunity and modulate the ability of DCs to present antigens and secrete cytokines (Gantier et al., 2007; Cheng et al., 2012; Smyth et al., 2015). For example, miR-24 has been shown to be regulated during macrophage and dendritic cell differentiation potentiates innate immunity (Fordham et al., 2015). MicroRNA146a reported to regulate human dendritic cell apoptosis and cytokine production by targeting TRAF6 and IRAK1 proteins (Park et al., 2015). Furthermore, AIV infection leads to the differential expression of cellular miRNA in chickens and mice, and miR491 and miR654 inhibit the replication of H1N1 virus through binding to PB1 in MDCK cells (Song et al., 2010). Since miRNA have the ability to modulate DC function, and our previous research work demonstrated that H9N2 AIV significantly influenced miRNA expression of DCs, the purpose of our study was to investigate the role miRNA play in regulating the immune response of DCs to PB1 stimulation.

\section{RESULTS}

\section{miRNA Expression following H9N2 AIV Infection and Viral Fragment Transfection}

To study how H9N2 might control miRNA expression, three segments of H9N2 AIV (PB1, PA, and NP) were cloned into pcDNA3.1 and transfected into bone marrow-derived DCs (BMDCs) (Supplementary Image 1). The expression of selected miRNAs was then examined by reverse transcription quantitative real-time PCR (RT-qPCR). Interestingly, for all of the miRNAs up-regulated by H9N2, PB1, PA, and NP significantly repressed their expression. For the miRNAs down-regulated by H9N2, the PB1 segment also mostly reduced their expression, especially for miR339, miR375, and miR146 (Figures 2A,B). Segments PB1, $\mathrm{PA}$, and NP are involved in the transcription and replication of the AIV RNA genome (Bouvier and Palese, 2008).

\section{Activation of Mouse BMDCs by PB1}

We first examined the phenotypic changes in BMDCs transfected with PB1, PA, and NP. The transfection efficiency of PB1, $\mathrm{PA}$, and NP was detected by RT-qPCR, and results are listed in Supplementary Data Sheet 1. Fluorescence-activated cell sorting (FACS) suggested that the mean fluorescence intensity (MFI) of MHCII was significantly enhanced by PB1, as were the co-stimulatory molecules CD40, CD80, and CD86 (Figures 3A,B). PA segment up-regulated CD80 and CD86, whilst NP had no effect on them (Figures 3A,B). Next, we assessed the ability of DCs to activate $\mathrm{T}$ lymphocytes and secrete cytokines. As shown in Figures 3C,D, PB1-stimulated DCs showed enhanced stimulation at a ratio of $1: 1$, and they expressed higher levels of interleukin-6 (IL-6) and tumor necrosis factor- $\alpha$ (TNF- $\alpha)$ than did the pcDNA3.1-transfected controls $(P<0.05)$. Whilst the LPS stimulation (positive control) were demonstrated to enhance the ability of DCs to present antigens, activate $\mathrm{T}$ lymphocytes, and secrete cytokines.

\section{The Immune Function of miR375 and miR181b in Regulating Mice BMDCs}

Recent studies have shown that miRNA regulate the immune responses of BMDCs (Wu et al., 2012). As H9N2 and PB1 significantly down-regulated the expression of miR375 (Figure 2), we examined the functions of miR375 in BMDCs. MiRNA over-expression vectors were constructed and validated by digesting with restriction enzyme Hind III and BamHI (Supplementary Image 2). FACS showed that miR375 overexpression decreased the percentage of CD80-, CD86-, CD40-, and MHCII when compared with the pSilencer4.1 group. While inhibition of miR375 greatly down-regulated the MFI of CD80 and CD86, but up-regulated the MFI of CD40 and MHCII when compared with the blank group (Figures 4A,B). Moreover, results also shown that the abundance of miR181b significant down-regulated all the surface -markers except MHC-II.). Interestingly, we found that the inhibition of miR181b significant increased the MFI of MHCII and CD-86 when compared with the blank group (Figures 4A,B). 


\section{Inhibition of Endogenous miR375 and miR181b Blocked PB1-Induced Phenotypic Alterations in BMDCs}

Previous studies demonstrated that PB1 and a number of miRNAs, including miR375 and miR181b, can influence the phenotype of BMDCs. Thus, we investigated whether the PB1induced changes in DCs are mediated by miRNAs. To test this hypothesis, miRNA inhibitors were added to DCs to repress endogenous miRNAs before PB1 transfection. FACS revealed that the inhibition of endogenous miR375 and miR181b decreased the expression of co-stimulatory molecules (CD80/CD86 and CD40) and MHCII, which was induced by PB1 $(P<0.05$; Figures 4 C,D).

\section{Effects on Signaling Pathways Stimulated by PB1, miR375 and miR181b}

Mitogen-activated protein kinase (MAPK) pathways exist in all eukaryotes and control a wide range of cellular processes, such as proliferation, differentiation, and survival (Kolch, 2000). We previously demonstrated that H9N2 AIV activates interferon (IFN) regulatory factor (IRF)-7. In this study, we first found that PB1 not only significant activated the p-Erk/Erk signaling pathway, but also inhibited the P-Jnk/Jnk signaling pathway when compared with the pcDNA3,1 group. Then, we found that over expressed miR375 significant decreased the expression level of $\mathrm{p}-\mathrm{IkBa} / \mathrm{IkBa}$ and IRF7, whilst the over expression of miR181b significant decreased the expression level of $\mathrm{p}-\mathrm{IkBa} / \mathrm{IkBa}$, IRF3 and IRF7, but significant increased the expression level of pErk/Erk. Furthermore, we found that the inhibition of miR375 hugely up-regulated the expression level of p-p38/p38, p-Erk/Erk and IRF7, but down-regulated the expression level of p-IkBa/ $\mathrm{IkBa}$ and $\mathrm{p}-\mathrm{Jnk} / \mathrm{Jnk}$ which suggested activation of P38, Erk, NF- $\mathrm{kB}$, and IFN signaling pathway. Finally, the inhibition of miR181b significant repressed the $\mathrm{p}$-Jnk/Jnk signaling pathway when compared with the blank group (Figures 5A,B).

\section{DISCUSSION}

The interactions between miRNA and DCs are important for AIV infection. In this report, we focused on miR375, which exhibited decreased expression in the PB1 segment stimulated group. DCs play an important role in the generation and maintenance of immune responses (Smyth et al., 2015). There are three standards for evaluating the immune function of BMDCs, including phenotypic alterations, the ability to activate T lymphocytes and the ability to secrete cytokines (Banchereau et al., 2000). Our study suggests that the immune function of DCs, including phenotypic alteration, T lymphocyte activation, and cytokine secretion, was greatly stimulated by PB1. Also, we demonstrated that expression of miR375 was suppressed by PB1.

MiRNAs repress key regulatory components of the innate immune response and markedly affected the capacity of DCs to present antigens and secrete cytokines. MicroRNA-375 was observed to influence cell proliferation, apoptosis and differentiation through the Notch signaling pathway, while microRNA-181b modulated the secretion of TNF- $\alpha$ and IL- $1 \beta$ in macrophages (Zhang et al., 2015; Wang et al., 2016). Our research suggests that increased expression of miR375 attenuated the DC immune responses induced by PB1. PB1 over-expression increased the levels of CD80-, CD86-, MHCII-, and CD40 in cultured BMDCs, whilst the inhibition of endogenous miR375 had the opposite effect (Figures 4C,D). MiR375 also modestly decreased the expression of CD80; this effect was reversed by inhibiting endogenous miR375. Also, miR181b repressed the expression of CD40 and MHCII; this inhibition effect was relieved when endogenous miR181b was silenced. All four surface markers are characteristics of fully mature DCs and represent different functionalities (Geissmann et al., 2010). The major histocompatibility complex class II (MHCII) are family of molecules normally found on antigen-presenting cells such as DCs and mononuclear phagocytes. The MHCII-dependent pathway of antigen presentation is called the exogenous pathway, which has been shown to be regulated by miRNAs (Tomasi et al., 2010). Over-expression of PB1 enhances the ability of DCs to express the surface markers, activate lymphocytes and secrete inflammatory cytokines. Whilst the addition of miR181b decreased the expression of MHCII and CD40, this effect can be repressed by inhibiting expression of miR181b. Thus, miR181b may enhance the function of DC by down-regulating surface maturation molecules MHCII. PB1 increased the expression of the pro-inflammatory cytokines IL- 6 and TNF- $\alpha$, but had no effect on the anti-inflammatory cytokines IL-10 and IL-12, which seem to trigger Th17 programming in the BMDC. DCs also promote Th1 responses via IL-12 (de Jong et al., 2002).

IFN- $\alpha$, controlled primarily by IFN regulatory factor 3 (IRF3 ) and IFN regulatory factor (IRF-7), plays a crucial role in host defense processes against viral infection (Taniguchi and Takaoka, 2002; Yanai and Taniguchi, 2008). H9N2 AIV infection could result in the activation of IRF-7 on DCs (Lin et al., 2014). Our results show that IRF-3 and IRF-7 were all down-regulated in miR375 and miR181b groups, while inhibition of endogenous miR375 and miR181b significantly decreases IRF-3 and IRF7, suggesting that miR375 and miR181b are necessary for the production of IFN- $\alpha$.

In summary, our results suggest that on the one hand, H9N2 virus protein $\mathrm{PB} 1$ can enhance the ability of DC to induce their phenotype, activate lymphocytes and secrete cytokines, and this effect may be accomplished by reducing the Jnk signaling pathway and activating the Erk signaling pathway. On the other hand, our results also suggest that miR375 can inhibit maturation of DC by decreasing expression of surface markers. Here, we demonstrated a previously unidentified role for PB1 in the regulation of murine immune responses of DCs, which was mediated by miR375 and miR181b. We propose that PB1 may enhance the function of DC by down-regulating miR375. Thus, miR375 may have a previously uncharacterised immunomodulatory role that can activate DCs for defense against H9N2 AIV (Figure 1).

\section{METHODS}

\section{Ethics Statement}

SPF C57BL/6 and BALB/c mice were obtained from Comparative Medical Center of Yang Zhou University. This study was 


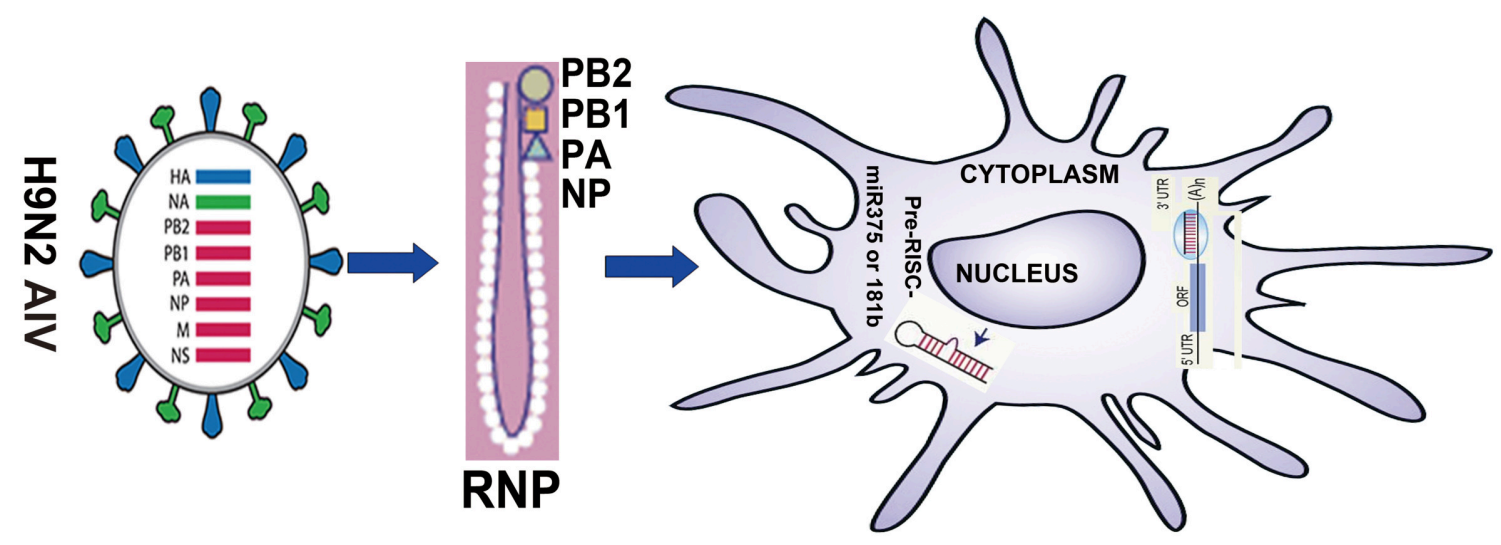

FIGURE 1 | Summary of the research strategy.
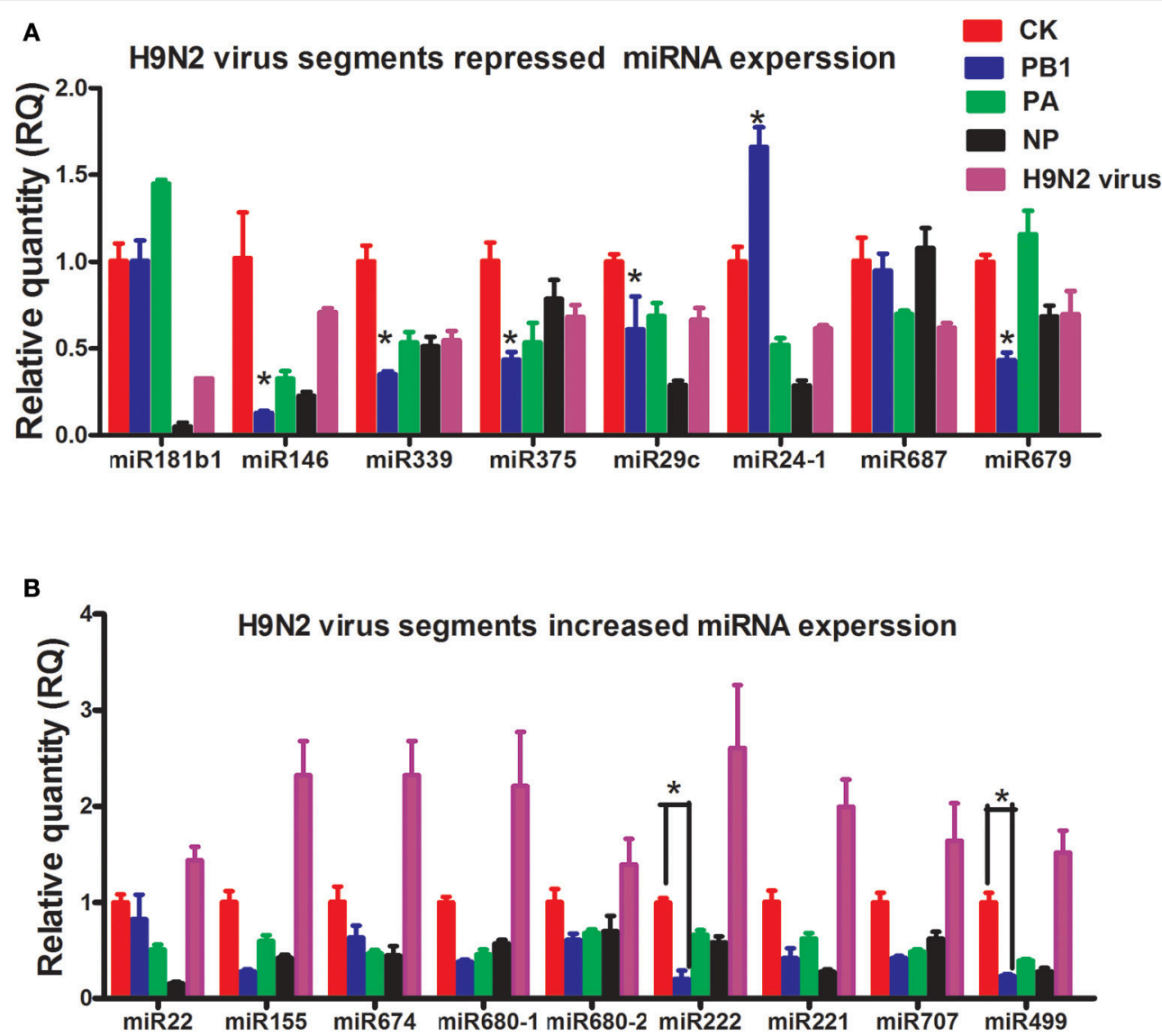

FIGURE 2 | Results of the qPCR analysis of select miRNAs following stimulation by PB1, PA, or NP. (A) The expression levels of down-regulated miRNAs stimulated by PB1, PA, or NP ( ${ }^{\star} P<0.01$, the significance of the data was determined by one-way ANOVA with Duncan test) (CK: blank DCs; PB1, PA, and NP: plasmid over-expressed DCs; LPS: $1 \mu \mathrm{g} / \mathrm{ml}$, each times, three 4-6 week wild-type male C57BL/6 mice were sacrificed to isolated BMDCs and experiments were performed at least in triplicate). (B) The expression levels of up-regulated miRNAs stimulated by PB1, PA, or NP ${ }^{\star} P<0.01$, the significance of the data was determined by one-way ANOVA with Duncan test) (CK: blank DCs; PB1, PA, and NP: plasmid over-expressed DCs; LPS: $1 \mu \mathrm{g} / \mathrm{ml})$.

approved by the Ethical Committee of Animal Experiments of the College of Veterinary Medicine, Nanjing Agricultural University. All animal care and use were conducted in strict accordance with the Animal Research Committee guidelines of the College of Veterinary Medicine, Nanjing Agricultural University. 
A
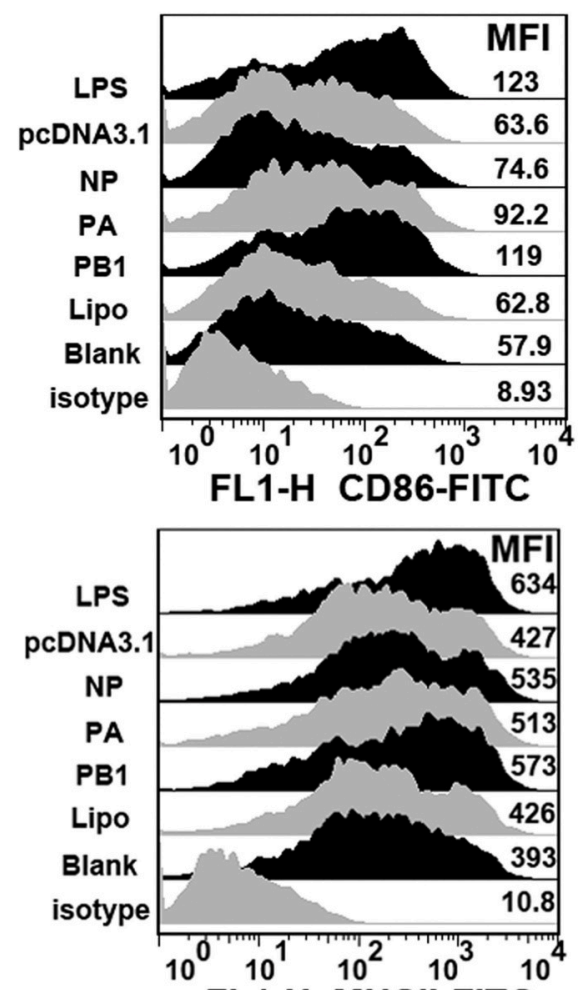

FL1-H MHCII-FITC

C
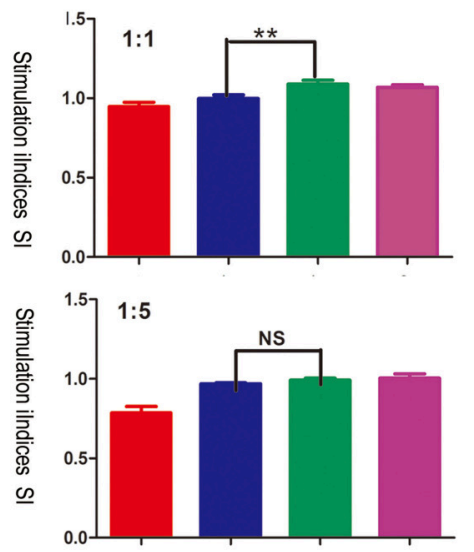

Blank $\square$ pcDNA3.1 PB1 $\square$ LPS

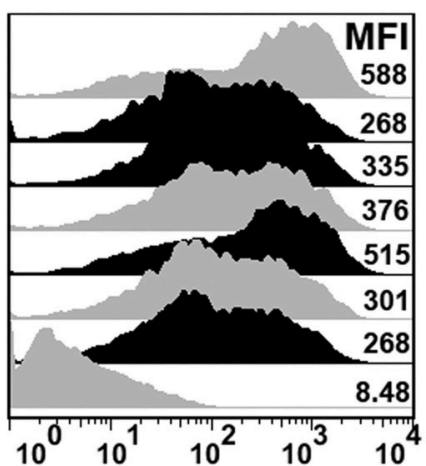

FL2-H CD80-PE

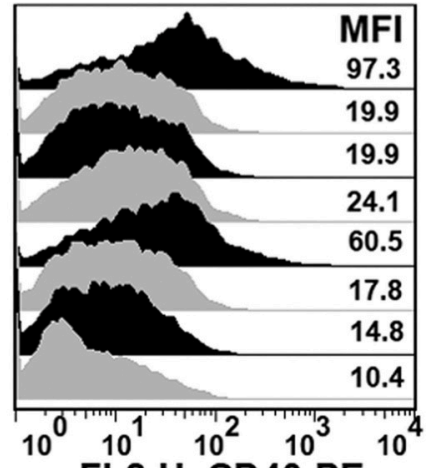

FL2-H CD40-PE
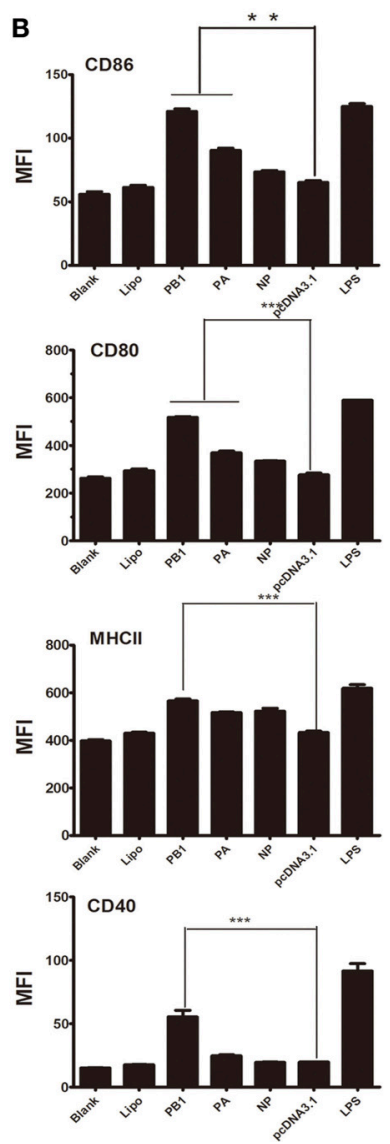

D
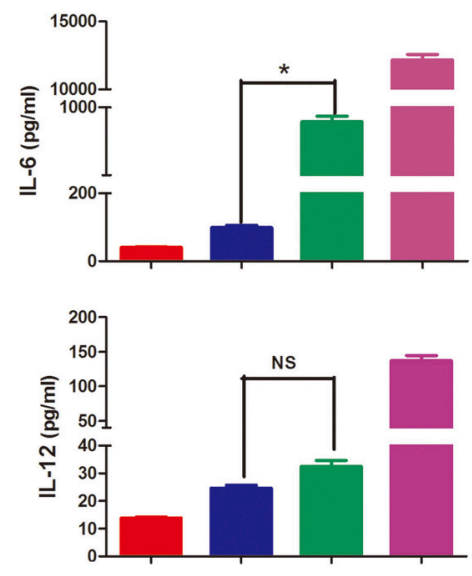

Blank
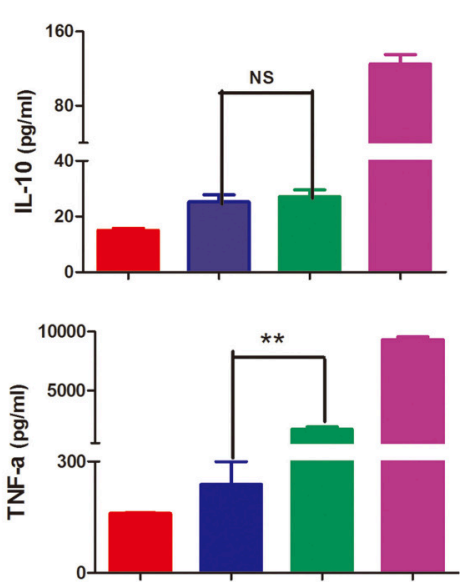

pcDNA3.1

PB1 LPS

FIGURE 3 | Immune activation of BMDCs stimulated by PB1. (A) Flow cytometric analysis of the phenotypic alterations in DCs stimulated with PB1, PA, or NP (i.e., the expressions of $\mathrm{CD} 40, \mathrm{CD} 80 / 86$, and $\mathrm{MHCll}$ on BMDCs stimulated with PB1, PA, or NP). (Isotype: IgG2a for CD40, IgG1 for CD80, and CD86, lgG2b for MHCIl; Blank: DCs without any treatment; Lipo: DCs added the same lipofectame as transfection groups; PB1, PA, and NP: plasmids transfected DCs; pcDNA3.1 group: plasmid pcDNA3.1 transfected DCs; LPS: Positive control, $1 \mu \mathrm{g} / \mathrm{ml}$ LPS, three 4-6 week wild-type male C57BL/6 mice were sacrificed to isolated BMDCs and experiments were performed at least in triplicate). (B) The MFI of CD40, CD80/86, and $\mathrm{MHCll}\left({ }^{\star} P<0.05,{ }^{\star \star} P<0.01\right.$, or ${ }^{\star \star \star} P<0.001$ the significance of the data was determined by one-way ANOVA with Tukey's multiple comparison test). (C) PB1-stimulated BMDCs stimulated the proliferation of naive T cells in mixed-lymphocyte reactions (MLR). The stimulator cells were BMDCs stimulated with or without PB1, pcDNA3.1, or LPS at $37^{\circ} \mathrm{C}$ for $24 \mathrm{~h}$. All experiments were performed at least in triplicate. Significant differences between the treated and pcDNA3.1 groups are expressed as ${ }^{\star} P<0.05$ or ${ }^{\star \star} P<0.01$. The significance of the data was determined by one-way ANOVA with Tukey's multiple comparison test. (D) Cytokine release from PB1-stimulated BMDCs was measured by enzyme-linked immunosorbent assays (ELISAs). Data for IL-6, IL-10, IL-12, and TNF- $\alpha$ are shown as means \pm standard deviation (SD) of three samples. Significant differences between the treated and pcDNA3.1 groups are expressed as ${ }^{*} P<0.05$ or ${ }^{* *} P<0.01$. The significance of the data was determined by one-way ANOVA with Tukey's multiple comparison test. 

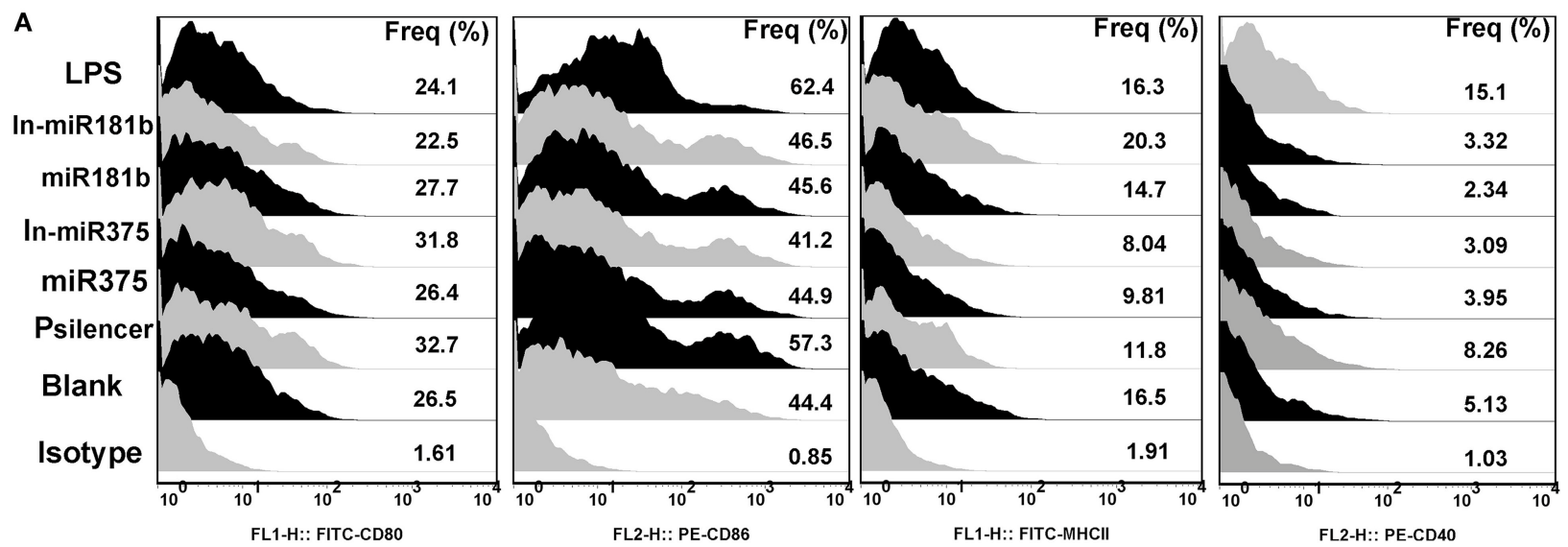

B
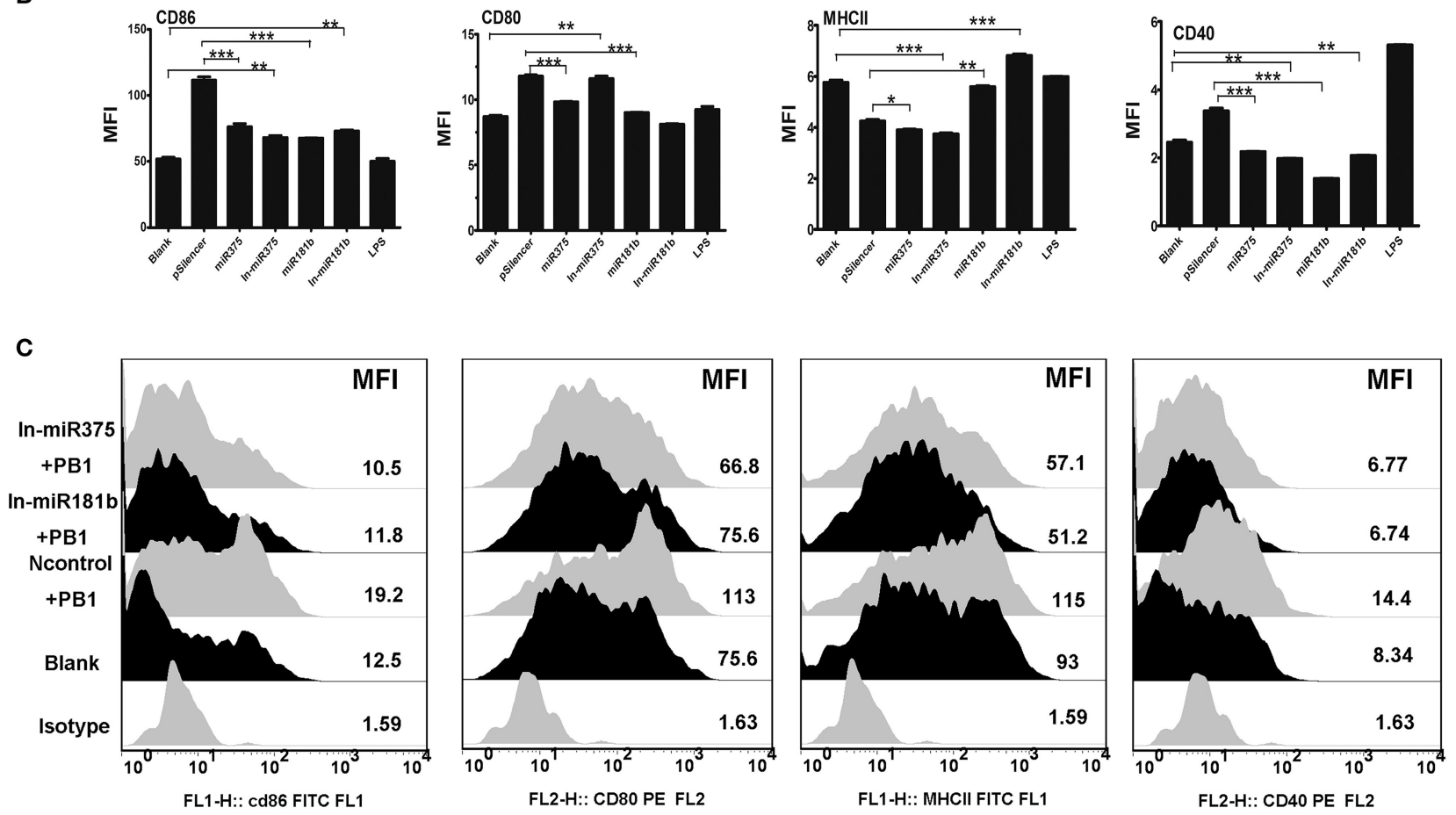

D
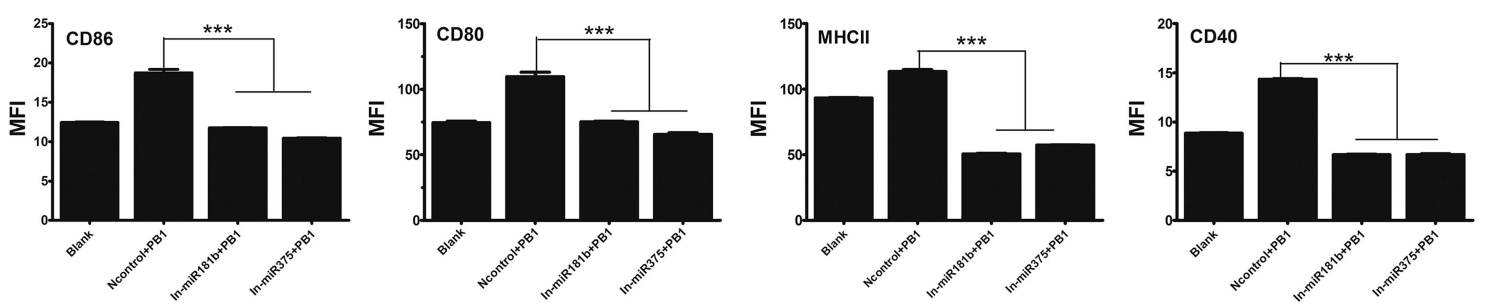

FIGURE 4 | The immune function of BMDCs stimulated by miR375 andmiR181b. (A) Flow cytometric analysis of the phenotypic alterations in DCs stimulated by miR375, miR181b, In-miR375, and In-miR181b (i.e., the expressions of CD40, CD80/86, and MHCII on BMDCs stimulated by miRNAs). (Isotype: IgG2a for CD40, IgG1 for CD80 and CD86, IgG2b for MHCll; Positive control, $1 \mu \mathrm{g} / \mathrm{ml} \mathrm{LPS}$, three 4-6 week wild-type male C57BL/6 mice were sacrificed to isolated BMDCs and experiments were performed at least in triplicate). (B) The MFI of CD40, CD80/86, and MHCll. ${ }^{\star} P<0.05$, ${ }^{\star \star} P<0.01$, or ${ }^{\star \star \star} P<0.001$ the significance of the data was determined by one-way ANOVA with Tukey's multiple comparison test). (C) PB1 mediated BMDCs activation when miR375 and miR181b were inhibited (100 $\mu \mathrm{g} / \mathrm{ml}$ inhibitor for each miRNA, Isotype: IgG2a for CD40, IgG1 for CD80 and CD86, IgG2b for MHCll, three 4-6 week wild-type male C57BL/6 mice were sacrificed to isolated BMDCs and experiments were performed at least in triplicate). (D) The MFI data for CD40, CD80/86, and MHCll. $\left({ }^{*} P<0.05\right.$, ${ }^{* *} P<0.01$, or ${ }^{* * *} P<0.001$ the significance of the data was determined by one-way ANOVA with Tukey's multiple comparison test). 


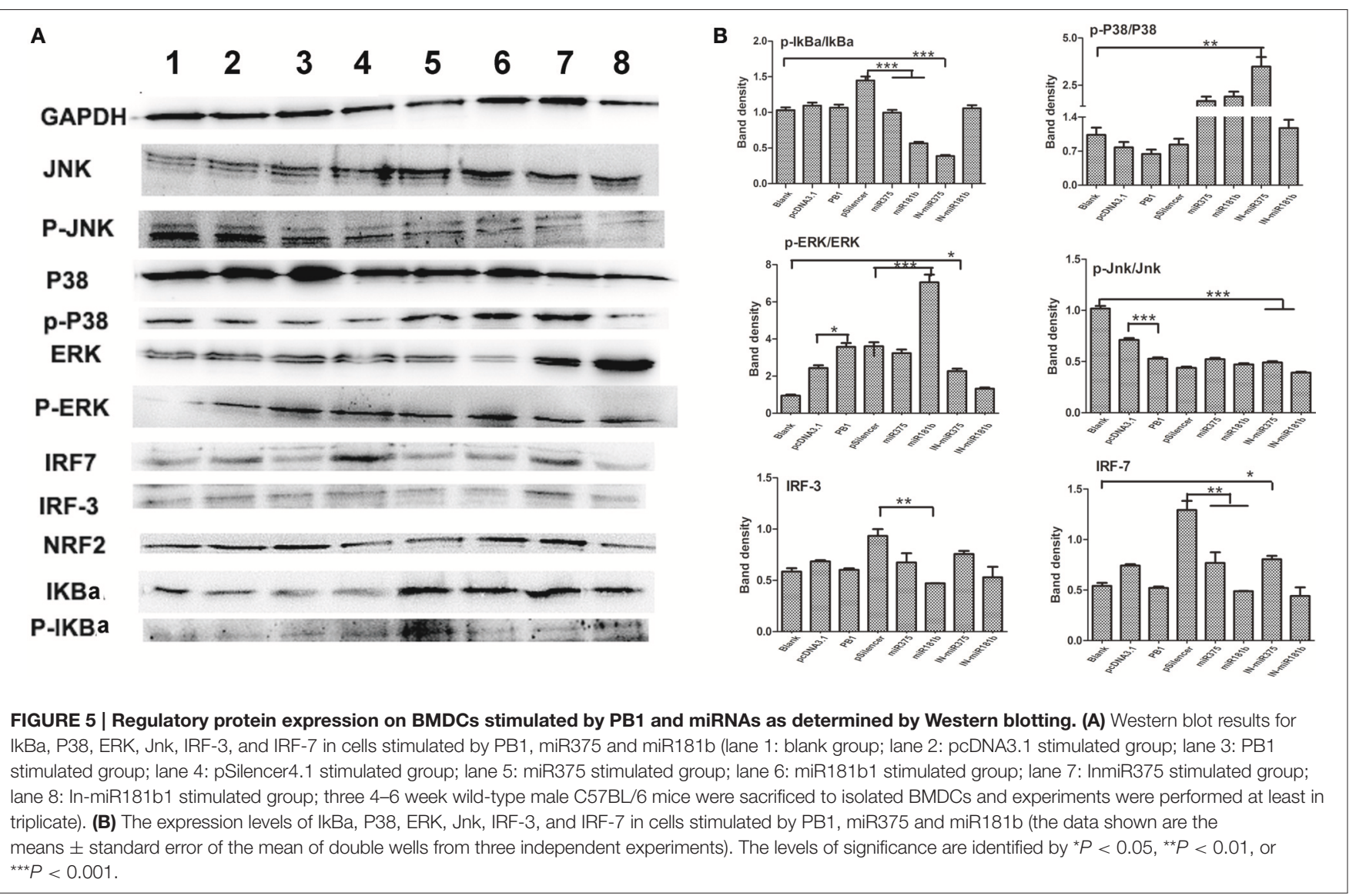

\section{Plasmids and Cell Culture}

Three RNA segments that encode proteins involved in viral replication (PB1, PA, and $\mathrm{NP}$ ) were amplified from the H9N2 virus and cloned into pcDNA3.1 (Invitrogen). MiRNAs (miR375 and miR-181) were amplified and cloned into pSilencer4.1 (Invitrogen). Bone marrow-derived dendritic cells (BMDCs) were prepared from the femurs and tibias of sacrificed 4-6 week wild-type male C57BL/6 mice and treated with red blood cell lysis buffer (Beyotime) (Lin et al., 2014). Briefly, bone marrow cells were flushed from the tibias and femurs and cultured in complete medium (RPMI1640 (Invitrogen) with 10\% FBS (Hyclone), 1\% streptomycin and penicillin, $10 \mathrm{ng} / \mathrm{ml}$ recombinant granulocyte-macrophage colony-stimulating factor (GM-CSF) and IL-4 (Peprotech) and plated in 6-well plates. At day 6, the non-adherent, relatively immature DCs $\left(1 \times 10^{6}\right.$ cells $/ \mathrm{ml}$ ) were harvested and centrifuged to remove debris and dead cells, then cultured overnight in complete medium and transfected with different plasmids for subsequent assays. Transferred cell samples $\left(1 \times 10^{6}\right.$ cells $)$ were washed twice with $\mathrm{PBS}$ and incubated at $4{ }^{\circ} \mathrm{C}$ for 30 min with the following monoclonal antibodies (anti-mouse CD11c (N418), anti-mouse CD40 (1C10), anti-mouse CD86 (GL1), anti-mouse MHC (major histocompatibility complex) class II (M5/114.15.2) and antimouse CD80 antibody (16-10A1), respectively (eBioscience). Finally, cells were analyzed using a Fluorescence Active Cell Sorter (FACS) (BD, FACS Aria) after two separate washes.

\section{The Choice of miRNAs and Quantitative PCR Validation}

To test which viral protein had the largest effect on expression of miRNAs, we amplified three replication related RNA segments (PB1, PA, and NP) and then cloned these into pcDNA3.1 vector. Primers are listed in Supplementary Table 1. Based on the microarray result, 9 up-regulated and 8 down-regulated genes were selected for RT-qPCR verification. Small RNAs were purified using the miRNeasy mini kit (Qiagen) and reverse transcribed to cDNA by miScript Reverse Transcriptase. QuantiTect SYBR Green PCR master mix (Qiagen) was used to perform $\mathrm{qPCR}$ according to the manufacturer's instructions. miRNA expression was normalized to the internal control 5S rRNA. Primers for 17 selected miRNAs are listed in Supplementary Table 2. All assays were performed in triplicate. Relative expression levels were calculated using the $2-\Delta \Delta \mathrm{Ct}$ method (Livak and Schmittgen, 2001).

\section{Immune Response of BMDCs Stimulated by PB1}

\section{Surface Marker Alterations of BMDCs}

Immature BMDCs were plated into fresh medium $\left(1 \times 10^{6}\right.$ cells $/ \mathrm{ml}$ ) and transfected with one of the three vector constructs(PB1, PA, and NP), pcDNA3.1 (negative control) and LPS ( $1 \mu \mathrm{g} / \mathrm{ml}$, positive control) for $48 \mathrm{~h}$. Then cell samples 
( $1 \times 10^{6}$ cells, $1.5 \mathrm{ml}$ tube) were collected, washed twice with PBS and incubated at $4{ }^{\circ} \mathrm{C}$ for $30 \mathrm{~min}$ with the following monoclonal antibodies (anti-mouse CD11c, anti-mouse CD40, anti-mouse CD86, anti-mouse MHC class II and anti-mouse CD80 antibody or the respective isotype controls, respectively). After washing, cells were analyzed with a Fluorescence Activated Cell Sorter (FACS) (BD, FACS Aria)

\section{Allogeneic Mixed Leukocyte Reaction (MLR) Proliferation Assays}

The primary T-cell stimulatory capacity of BMDCs was examined in a MLR. Untreated and variously treated BMDCs [pcDNA3.1stimulated, PB1-stimulated and LPS-stimulated $(1 \mu \mathrm{g} / \mathrm{ml})]$ were used as the stimulator cells. Allogeneic lymphocytes were obtained from BALB/c mice as follows. Leukocytes were isolated from the spleens of 4 to 6 week-old allogeneic BALB/c mice with a T cell isolation kit (Miltenyi, Bergisch Gladbach, Germany) and cultured in complete RPMI 1640 medium supplementaryed with $10 \%$ FCS in 96 -well plates at $37^{\circ} \mathrm{C}$ for $48 \mathrm{~h}$. Graded numbers of responder cells $\left(1 \times 10^{5}\right.$ cells/well $)$ were added to 96-well round bottomed plates, giving responder: stimulator ratios of 1:1 or $5: 1$, in a culture volume of $100 \mu \mathrm{l}$. Cell proliferation assays was conducted with the Cell Counting Kit-8 (CCK-8, Beyotime). Each well received $20 \mu \mathrm{l}$ CCK- 8 solution and was incubated for a further $2 \mathrm{~h}$ at $37^{\circ} \mathrm{C}$ before absorbance measurement at $450 \mathrm{~nm}$. All experiments were conducted in triplicate. The Stimulation Index was calculated using the formula:

$$
\begin{aligned}
\mathrm{SI}= & \left(\mathrm{OD}_{\text {sample }}-\mathrm{OD}_{\text {stimulator cells only }}\right) / \\
& \left(\mathrm{OD}_{\text {responder cells only }}-\mathrm{OD}_{\text {blankcontrol }}\right)
\end{aligned}
$$

\section{Cytokine Analysis}

Bone marrow-derived dendritic cells (BMDC) culture supernatants were collected at $24 \mathrm{~h}$ after treatments (Groups were divided as MLR experiments). Concentrations of TNF- $\alpha$, IL-6, IL-10, and IL-12 $p_{70}$ in the supernatants were measured using the Quantikine Elisa kit (Boster) according to the manufacturer's instructions. The sensitivity of the assay was $2 \mathrm{pg} / \mathrm{ml}$ for TNF- $\alpha$, and $4 \mathrm{pg} / \mathrm{ml}$ for IL-6, IL-10, and IL-12 $\mathrm{p}_{70}$.

\section{qRT-PCR Validation}

BMDCs was cultured and collected at $24 \mathrm{~h}$ after treatments with PB1, PA and NP segments. qPCR was conducted to examine expression variation for selected genes. Individual samples were diluted $1: 5$ and $2 \mu \mathrm{l}$ was amplified in a $20 \mu \mathrm{l}$ reaction containing $10 \mu l$ of SYBR Premix ${ }^{\mathrm{TM}}$ Ex Taq (TaKaRa), $0.4 \mu$ l of ROX dye II and $0.4 \mu \mathrm{M}$ of each of the forward and reverse gene-specific primers using an ABI 7500 instrument (Applied Biosystems, USA). - We also evaluated the transcription efficiency of plasmid pcDNA3.1-PA, NP, and PB1 by qPCR. Primers are- listed in Supplementary Table 3.

\section{Immune Response of BMDCs Stimulated with miRNAs}

\section{Plasmid Construction and Phenotypic Detection}

To confirm that the phenotype alteration induced by PB1 is meditated by miRNA, miRNA over-expression vectors were constructed using the pSilencer4.1 vector (Invitrogen). Four selected miRNAs (miR375 and miR181b) were amplified and then cloned into pSilencer4.1. Primers are listed in Supplementary Table 4. The isolation of BMDCs and phenotypic detection were performed as described above Plasmids were transfected with lipofectame2000 reagent (Invitrogen). MiR181b and miR375 inhibitors, which were chemically modified single stranded RNAs, were designed and purchased from RiboBio to evaluate miRNA function (Guangzhou, China). Each $100 \mathrm{nM}$ miRNA inhibitor (micrOFF $^{\mathrm{TM}}$ mmu-miR-181b-3p inhibitor, micrOFF ${ }^{\mathrm{TM}} \mathrm{mmu}-$ miR-375-5p inhibitor, and micrOFF $^{\mathrm{TM}}$ inhibitor Negative Control) was transfected into BMDCs for $24 \mathrm{~h}$ to analyze their effect on DCs via detection of- phenotypic alteration with FACS.

\section{miRNA Inhibition Experiment}

To detect whether the phenotypic alteration of BMDCs induced by PB1 was mediated by miR181b or miR375, miRNAs inhibitors were transfected into BMDCs for $4 \mathrm{~h}$ as described above, before PB1 over-expression plasmid was transfected. After another $24 \mathrm{~h}, \mathrm{BMDCs}$ were collected for phenotypic detection using FACS.

\section{Western Blot Assay}

MAPK, NF-kB, and IFN-a signaling pathways control a wide range of cellular processes, especially for the immune response. Thus, we tried to evaluated how PB1 and their induced miRNAs affect the MAPK, NF-kB, and IFN-a signal pathways by western blot. BMDCs were transfect with PB1, miR375, miR181b, InmiR375, and In-miR181b for $48 \mathrm{~h}$. Then cells were collected and washed with PBS three times for the next experiments. Western blot detection was performed as previously described by us. Mouse IkBa, P-IkBa, P38, P-P38, ERK, P-ERK, JUK, P-JUK, IRF-3, and IRF-7 were purchased from Abcam or Cell Signaling Technology and detected according to each manufacturer's protocol. Protein bands were visualized using the Super ECL Plus system. GAPDH was used as a loading control (Abcam).

\section{Statistical Analyses}

Data were evaluated by unpaired two-tailed Student's $t$-test using GraphPad Prism 5 (http://www.graphpad.com) (CSSN), with $p<0.05$ considered to be statistically significant. The significance of the data was also determined by one-way ANOVA, followed by Tukey's multiple comparison tests. FACS data were analyzed by FlowJo software (FlowJo, China). All data are expressed as mean \pm standard error of the mean.

\section{AUTHOR CONTRIBUTIONS}

JL design and performed all the experiments, analyzed the data and drafted the manuscript, JX and CT developed the dendritic cells and performed flow cytometry analyses, $\mathrm{KZ}$ charged for the data analyzed, YZ and QY supervised the experiment and participated in the design. All the authors read and approved the final manuscript. 


\section{ACKNOWLEDGMENTS}

This work was supported by the National Natural Science Foundation of China (No. 31172302), the Natural Science Foundation of Jiangsu Province (No. BK20150666), the National Natural Science Foundation of China (No. 31570843) and A Project Funded by the Priority Academic Program Development of Jiangsu Higher Education Institutions (PAPD).

\section{SUPPLEMENTARY MATERIAL}

The Supplementary Material for this article can be found online at: http://journal.frontiersin.org/article/10.3389/fmicb. 2017.00287/full\#supplementary-material

Supplementary Image 1 | Identification and construction of pcDNA3.1-PB1, pcDNA3.1-PA, and pcDNA3.1-NP. (A) Identification of pcDNA3.1-PB1 by digestion with Xhol and Kpnl (M1: DL5000 DNA marker; 1: plasmid pcDNA3.1-PB1; 2: plasmid pcDNA3.1-PB1 digested with Xhol and Kpnl). (B) Identification of pcDNA3.1-NP by digestion with Xhol and Hindlll (M1: DL5000 DNA marker; 1: plasmid pcDNA3.1-NP; 2: plasmid pcDNA3.1-NP digested with

\section{REFERENCES}

Banchereau, J., Briere, F., Caux, C., Davoust, J., Lebecque, S., Liu, Y. J., et al. (2000). Immunobiology of dendritic cells. Annu. Rev. Immunol. 18, 767-811. doi: 10.1146/annurev.immunol.18.1.767

Bouvier, N. M., and Palese, P. (2008). The biology of influenza viruses. Vaccine 26(Suppl. 4), D49-D53.

Cheng, J. C., Yeh, Y. J., Tseng, C. P., Hsu, S. D., Chang, Y. L., Sakamoto, N., et al. (2012). Let-7b is a novel regulator of hepatitis C virus replication. Cell. Mol. Life Sci. 69, 2621-2633. doi: 10.1007/s00018-012-0940-6

de Jong, E. C., Vieira, P. L., Kalinski, P., Schuitemaker, J. H., Tanaka, Y., Wierenga, E. A., et al. (2002). Microbial compounds selectively induce Th1 cell-promoting or Th2 cell-promoting dendritic cells in vitro with diverse th cell-polarizing signals. J. Immunol. 168, 1704-1709. doi: 10.4049/jimmunol.168.4.1704

Fang, L. Q., Li, X. L., Liu, K., Li, Y. J., Yao, H. W., Liang, S., et al. (2013). Mapping spread and risk of avian influenza A (H7N9) in China. Sci. Rep. 3:2722. doi: 10.1038/srep02722

Fordham, J. B., Naqvi, A. R., and Nares, S. (2015). Regulation of miR24, miR-30b, and miR-142-3p during macrophage and dendritic cell differentiation potentiates innate immunity. J. Leukoc. Biol. 98, 195-207. doi: 10.1189/jlb.1A1014-519RR

Gantier, M. P., Sadler, A. J., and Williams, B. R. (2007). Fine-tuning of the innate immune response by microRNAs. Immunol. Cell Biol. 85, 458-462. doi: 10.1038/sj.icb.7100091

Geissmann, F., Manz, M. G., Jung, S., Sieweke, M. H., Merad, M., and Ley, K. (2010). Development of monocytes, macrophages, and dendritic cells. Science 327, 656-661. doi: 10.1126/science.1178331

Hemerka, J. N., Wang, D., Weng, Y., Lu, W., Kaushik, R. S., Jin, J., et al. (2009). Detection and characterization of influenza A virus PA-PB2 interaction through a bimolecular fluorescence complementation assay. J. Virol. 83, 3944-3955. doi: 10.1128/JVI.02300-08

Isakova-Sivak, I., Korenkov, D., Smolonogina, T., Tretiak, T., Donina, S., Rekstin, A., et al. (2017). Comparative studies of infectivity, immunogenicity and cross-protective efficacy of live attenuated influenza vaccines containing nucleoprotein from cold-adapted or wild-type influenza virus in a mouse model. Virology 500, 209-217. doi: 10.1016/j.virol.2016.10.027

Jin, Y., Yu, D., Ren, H., Yin, Z., Huang, Z., Hu, M., et al. (2014). Phylogeography of Avian influenza A H9N2 in China. BMC Genomics. 15:1110. doi: 10.1186/1471-2164-15-1110
Xhol and HindllI). (C) Identification of pcDNA3.1-PA by digestion with Xhol and HindIII (M1: DL5000 DNA marker; 1-3: plasmid pcDNA3.1-PA digested with Xhol and HindIII; 4: plasmid pcDNA3.1-PA)

\section{Supplementary Image 2 | Identification and construction of} pSilencer-miR375 and pSilencer-miR181b by digestion with BamHI and HindIII. (A) Identification of pSilencer-miR181b by digestion with $\mathrm{BamHI}$ and HindIII (M1: DL5000 DNA marker; 1: plasmid pSilencer- miR181b; 2: pSilencer-miR181b digested with BamHI and HindllI). (B) Identification of pSilencer-miR375 by digestion with BamHI and HindIII (M1: DL5000 DNA marker; 1: pSilencer-miR375 digested with BamHI and HindIII; 2: plasmid pSilencer-miR375).

Supplementary Table 1 | Primers used in amplified PB1, PA and NP.

Supplementary Table 2 | qRT-PCR primers used for detecting miRNAs alteration.

Supplementary Table 3 | qRT-PCR primers used for detecting target genes and viral segments.

Supplementary Table 4 | Primers used in amplified miR375 and miR181b1.

Supplementary Data Sheet 1 | Results of the qPCR analysis of the transfection efficient on BMDCs with plasmid pcDNA3.1-PA, pcDNA3.1-NP and pcDNA3.1-PB1.

Kolch, W. (2000). Meaningful relationships: the regulation of the Ras/Raf/MEK/ERK pathway by protein interactions. Biochem. J. 351, 289-305. doi: 10.1042/bj3510289

Liang, J., Fu, J., Kang, H., Lin, J., Yu, Q., and Yang, Q. (2013). The stimulatory effect of TLRs ligands on maturation of chicken bone marrow-derived dendritic cells. Vet. Immunol. Immunopathol. 155,205-210. doi: 10.1016/j.vetimm.2013.06.014

Lin, J., Yin, Y. Y., Qin, T., Zhu, L. Q., Yu, Q. H., and Yang, Q. (2014). Enhanced immune response of BMDCs pulsed with $\mathrm{H} 9 \mathrm{~N} 2 \mathrm{AIV}$ and $\mathrm{CpG}$. Vaccine 32,6783-6790. doi: 10.1016/j.vaccine.2014.10.013

Livak, K. J., and Schmittgen, T. D. (2001). Analysis of relative gene expression data using real-time quantitative PCR and the $2^{-\Delta \Delta \mathrm{C}_{\mathrm{T}}}$ Method. Methods 25, 402-408. doi: 10.1006/meth.2001.1262

Lopez, C. B., Moltedo, B., Alexopoulou, L., Bonifaz, L., Flavell, R. A., and Moran, T. M. (2004). TLR-independent induction of dendritic cell maturation and adaptive immunity by negative-strand RNA viruses. J. Immunol. 173, 6882-6889. doi: 10.4049/jimmunol.173.11.6882

Massari, S., Goracci, L., Desantis, J., and Tabarrini, O. (2016). Polymerase Acidic Protein-Basic Protein 1 (PA-PB1) Protein-Protein Interaction as a Target for Next-Generation Anti-influenza Therapeutics. J. Med. Chem. 59, 7699-7718. doi: 10.1021/acs.jmedchem.5b01474

O'Donnell, C. D., and Subbarao, K. (2011). The contribution of animal models to the understanding of the host range and virulence of influenza A viruses. Microbes Infect. 13, 502-515. doi: 10.1016/j.micinf.2011.01.014

Park, H., Huang, X., Lu, C., Cairo, M. S., and Zhou, X. (2015). MicroRNA-146a and microRNA-146b regulate human dendritic cell apoptosis and cytokine production by targeting TRAF6 and IRAK1 proteins. J. Biol. Chem. 290, 2831-2841. doi: 10.1074/jbc.M114.591420

Peiris, M., Yuen, K. Y., Leung, C. W., Chan, K. H., Ip, P. L., Lai, R. W., et al. (1999). Human infection with influenza H9N2. Lancet 354, 916-917.

Pu, J., Wang, S., Yin, Y., Zhang, G., Carter, R. A., Wang, J., et al. (2015). Evolution of the H9N2 influenza genotype that facilitated the genesis of the novel H7N9 virus. Proc. Natl. Acad. Sci. U.S.A. 112, 548-553. doi: 10.1073/pnas. 1422456112

Shaib, H. A., Cochet, N., Ribeiro, T., Abdel Nour, A. M., Nemer, G., Azhar, E., et al. (2014). Passaging impact of H9N2 avian influenza virus in hamsters on its pathogenicity and genetic variability. J. Infect. Dev. Ctries. 8, 570-580. doi: $10.3855 /$ jidc. 4023

Smyth, L. A., Boardman, D. A., Tung, S. L., Lechler, R., and Lombardi, G. (2015). MicroRNAs affect dendritic cell function and phenotype. Immunology 144, 197-205. doi: 10.1111/imm.12390 
Song, L., Liu, H., Gao, S., Jiang, W., and Huang, W. (2010). Cellular microRNAs inhibit replication of the H1N1 influenza A virus in infected cells. J. Virol. 84, 8849-8860. doi: 10.1128/JVI.00456-10

Swale, C., Monod, A., Tengo, L., Labaronne, A., Garzoni, F., Bourhis, J. M., et al. (2016). Structural characterization of recombinant IAV polymerase reveals a stable complex between viral PA-PB1 heterodimer and host RanBP5. Sci. Rep. 6:24727. doi: 10.1038/srep24727

Taniguchi, T., and Takaoka, A. (2002). The interferon-alpha/beta system in antiviral responses: a multimodal machinery of gene regulation by the IRF family of transcription factors. Curr. Opin. Immunol. 14, 111-116. doi: 10.1016/S0952-7915(01)00305-3

Tomasi, T. B., Magner, W. J., Wiesen, J. L., Oshlag, J. Z., Cao, F., Pontikos, A. N., et al. (2010). MHC class II regulation by epigenetic agents and microRNAs. Immunol. Res. 46, 45-58. doi: 10.1007/s12026-0098128-3

Tucci, M., Stucci, S., Passarelli, A., Giudice, G., Dammacco, F., and Silvestris, F. (2014). The immune escape in melanoma: role of the impaired dendritic cell function. Expert Rev. Clin. Immunol. 10, 1395-1404. doi: 10.1586/1744666X. 2014.955851

Wang, L., Song, G., Liu, M., Chen, B., Chen, Y., Shen, Y., et al. (2016). MicroRNA-375 overexpression influences P19 cell proliferation, apoptosis and differentiation through the Notch signaling pathway. Int. J. Mol. Med. 37, 47-55. doi: 10.3892/ijmm.2015.2399

Wu, C., Gong, Y., Yuan, J., Zhang, W., Zhao, G., Li, H., et al. (2012). microRNA-181a represses ox-LDL-stimulated inflammatory response in dendritic cell by targeting c-Fos. J. Lipid Res. 53, 2355-2363. doi: 10.1194/jlr. M028878

Yanai, H., and Taniguchi, T. (2008). [IRF family transcription factors and host defense signaling]. Tanpakushitsu Kakusan Koso 53, 1231-1238.

Zhang, Y., Wang, F., Lan, Y., Zhou, D., Ren, X., Zhao, L., et al. (2015). Roles of microRNA-146a and microRNA-181b in regulating the secretion of tumor necrosis factor- $\alpha$ and interleukin- $1 \beta$ in silicon dioxide-induced NR8383 rat macrophages. Mol. Med. Rep. 12, 5587-5593. doi: 10.3892/mmr.2015.4083

Zhou, P., Zhu, W., Gu, H., Fu, X., Wang, L., Zheng, Y., et al. (2014). Avian influenza H9N2 seroprevalence among swine farm residents in China. J. Med. Virol. 86, 597-600. doi: 10.1002/jmv.23869

Zhou, Z., Li, X., Liu, J., Dong, L., Chen, Q., Kong, H., et al. (2015). Honeysuckleencoded atypical microRNA2911 directly targets influenza A viruses. Cell Res. 25, 39-49. doi: 10.1038/cr.2014.130

Conflict of Interest Statement: The authors declare that the research was conducted in the absence of any commercial or financial relationships that could be construed as a potential conflict of interest.

Copyright (c) 2017 Lin, Xia, Tu, Zhang, Zeng and Yang. This is an open-access article distributed under the terms of the Creative Commons Attribution License (CC BY). The use, distribution or reproduction in other forums is permitted, provided the original author(s) or licensor are credited and that the original publication in this journal is cited, in accordance with accepted academic practice. No use, distribution or reproduction is permitted which does not comply with these terms. 